\section{Therapie älterer AML-Patienten}

\begin{abstract}
Ältere Menschen haben das höchste Risiko für die Entwicklung der zwar seltenen, aber mit einer schlechten Prognose verbundenen akuten myeloischen Leukämie (AML). Altersbedingte Faktoren wie Komorbiditäten, eine eingeschränkte Performance und krankheitsbedingte ungünstige Prognosefaktoren limitieren die Therapieoptionen.
\end{abstract}

Bistor isher existieren keine allgemein akzeptierten Therapieleitlinien für AML-Patienten, die älter als 60 Jahre sind. Das US-amerikanische National Comprehensive Cancer Network (NCCN) empfiehlt eine standardmäßige Induktionschemotherapie (IC) für über 60-Jährige mit AML und günstigen Prognosefaktoren. Patienten, die diese Kriterien nicht aufweisen, erhalten gewöhnlich niedrigdosiertes Cytarabin oder ausschließlich unterstützende Behandlungsmaßnahmen. Die medianen Überlebenszeiten liegen für diese beiden Optionen bei nur 5 bzw. 2 Monaten. Das NCCN rät für diesen problematischen Patientenkreis auch zu einer Therapie auf Basis des hypomethylierenden Agens Azacitidin, mit dem sich in früheren Studien ermutigende Gesamtüberlebensraten erzielen ließen.

Jetzt liegen die Ergebnisse der internationalen, multizentrischen Phase-IIIStudie „AZA-AML-001“ vor, in der erstmals prospektiv die Wirksamkeit und Sicherheit von Azacitidin im Vergleich zu konventionellen Behandlungsregimes (CCR) evaluiert wurden. An der Studie hatten 488 Patienten im Alter von $\geq 65$ Jahren mit einer neu diagnostizierten AML und einem Blastenanteil im Knochenmark von über $30 \%$ teilgenommen. Vor der Randomisierung in 2 gleich große Therapiearme wurde jeder Patient für eine CCR (Standard-IC, niedrigdosiertes Cytarabin oder ausschließlich supportive Maßnahmen) vorselektiert.

Die Patienten erhielten entweder $75 \mathrm{mg} / \mathrm{m}^{2}$ Azacitidin subkutan (s. c.) an 7 aufeinanderfolgenden Tagen eines 28-tägigen Zyklus (mindestens 6 Zyklen) oder das vorgesehene konventionelle Regime. Patienten in der Azacitidin-Gruppe überlebten mit median 10,4 gegenüber 6,5 Monaten länger als die konventionell Behandelten. Azacitidin verbesserte auch die 1-Jahres-Gesamtüberlebensrate (46,5 vs. $34,2 \%$ ). Die unerwünschten $\mathrm{Ne}$ - benwirkungen blieben innerhalb des für Azacitidin etablierten Sicherheitsprofils. Patienten aller Subgruppen profitierten von der Azacitidin-Behandlung.

Fazit: Azacitidin könnte sich als zusätzliche Therapieoption für ältere Patienten mit einer neu diagnostizierten AML etablieren. Hinweise auf positive Effekte von Kombinationen mit Lenalidomid, Panobinostat oder Sorafenib bedürfen einer Bestätigung in weiterführenden Studien. Wolfgang Zimmermann

Dombret $\mathrm{H}$ et al. International phase 3 study of azacitidine vs conventional care regimens in older patients with newly diagnosed AML with > 30\% blasts. Blood. 2015;126(3):291-9.

\section{Kommentar von Prof. Wäsch}

Die meisten Patienten, die an einer AML erkranken, sind älter als 60 Jahre. Gleichzeitig geht die AML bei älteren Patienten oft aus einem myelodysplastischen Syndrom (MDS) hervor oder hat einen ungünstigen Karyotyp. Im Gegensatz zu älteren Patienten mit günstiger Zytogenetik profitieren diese Patienten wenig von einer konventionellen Induktionstherapie, da das Ansprechen schlecht oder von kurzer Dauer ist und gleichzeitig eine hohe Toxizität besteht. Eine Heilung kann bei diesen Patienten in der Regel nur durch eine allogene Stammzelltransplantation (SCT) erreicht werden. Deshalb sind besser verträgliche Therapiealternativen dringend erforderlich, entweder als definitive Therapie, die ein besseres Überleben ermöglicht als die konventionelle Induktion, niedrigdosiertes Cytarabin oder "Best Supportive Care" oder als Überbrückung zu einer SCT mit reduzierter Konditionierung.

Bereits zugelassen war die hypomethylierende Substanz Azacitidin für Patienten mit MDS, chronischer myelomonozytärer Leukämie (CMML) und AML mit 20-30\% Blasten. In der aktuellen Phase-III-Studie von Hervé Dombret und Kollegen mit Patien- ten im Alter von 65 Jahren und darüber zeigt sich jetzt ein längeres Gesamtüberleben unter Azacitidin auch bei der AML mit mehr als $30 \%$ Blasten. Dies führte zur Zulassung des Medikaments auch für diese Indikation. Damit etabliert sich neben Decitabin eine weitere hypomethylierende Substanz zur Therapie der AML mit einem verbesserten Gesamtüberleben gegenüber der konventionellen Therapie, einem günstigen Nebenwirkungsprofil und einfacher subkutaner Anwendung sowie ambulanter Therapiedurchführung.

Da das Ansprechen auf hypomethylierende Substanzen langsam erfolgt, ist zur Beurteilung eine Behandlung mit mehreren Zyklen erforderlich, bevor das Ansprechen beurteilt werden kann. In der Studie von Dombret und Kollegen wurden deshalb mindestens 6 Zyklen geplant.

Obwohl Azacitidin die Therapieoptionen erweitert, handelt es sich nicht um eine kurative Therapie. Deshalb müssen Kombinationen von Azacitidin mit zielgerichteten Therapien wie Sorafenib, Volasertib u.a. getestet werden. Die Kombination von Azacitidin und Volasertib soll bei MDS/ CMML in einer Phase-I-Studie in Deutschland geprüft werden und stellt einen vielversprechenden Ansatz auch für die AML

\section{„Möglicherweise könnte Azacitidin als ,Bridging' zur allogenen Transplantation dienen."}

dar. Ferner könnte der Einsatz des toxizitätssparenden Azacitidins als Überbrückung bis zur allogenen SCT mit reduzierter Konditionierung eine kurative Option für ausgewählte Patienten eröffnen. In einer Multicenter-Studie der EORTC wird derzeit in einem solchen Konzept Decitabin mit konventioneller Induktion und anschliessender allogener SCT verglichen. Wichtig ist, für geeignete Patienten frühzeitig einen Spender zu suchen und sie rechtzeitig in einem Transplantationszentrum vorzustellen.

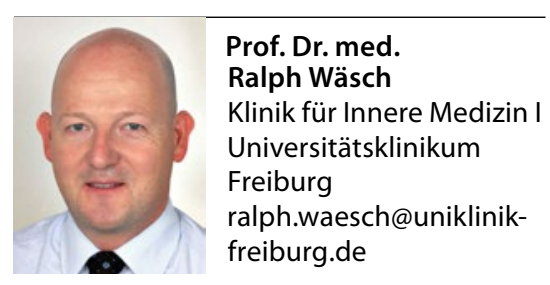

